\title{
DISEÑO Y ESTABLECIMIENTO DE UN HUERTO SEMILLERO CLONAL DE GENERACIÓN AVANZADA DE Nothofagus obliqua (Mirb.) Oerst
}

\author{
Ipinza, Roberto ${ }^{1}$; Gutiérrez, Braulio²; Clasing, Germán ${ }^{3}$; \\ Corti, Dante ${ }^{4}$ y Molina, Maria Paz
}

\section{RESUMEN}

Se describe el trabajo de diseño y plantación de un huerto semillero clonal de segunda generación de roble (Nothofagus obliqua), establecido en julio de 2016 en Huillilemu (San José de la Mariquina, región de Los Ríos, Chile).

El principal objetivo del trabajo es constituir una referencia u orientación para otras iniciativas similares que busquen maximizar las ganancias genéticas y minimizar la endogamia bajo restricciones de parentesco.

También se presentan y discuten consideraciones claves para enfrentar las decisiones involucradas en el diseño de huertos semilleros de generación avanzada.

Palabras clave: Huerto semillero, diseño, endogamia, ganancia genética

\section{SUMMARY}

A second generation roble (Nothofagus obliqua) clonal seed orchard design and planting is described. The orchard was established in July 2016 at Huillilemu farm (San José de la Mariquina, Los Rios region, Chile).

Main work objective is to be a guidance for other similar initiatives that seek to maximize gains and to minimize genetic inbreeding under restrictions of relative relationship.

Key considerations to address the decisions involved in designing advanced generation seed orchard are discussed too.

Keywords: Seed orchard, design, inbreeding, genetic gain.

\footnotetext{
${ }_{1}^{1}$ Ingeniero Forestal. Dr. Ingeniero de Montes. Instituto Forestal, sede Los Ríos. roberto.ipinza@infor.cl

2 Ingeniero Forestal. Instituto Forestal, sede Biobío. bgutierr@infor.cl

${ }^{3}$ Ingeniero Forestal. Corporación Nacional Forestal, Los Ríos.german.clasing@conaf.cl

${ }^{4}$ Ingeniero Forestal. M.Sc., Dr. en Tecnologías de Información Geográfica. Instituto Forestal, sede Los Ríos. dcorti@infor.cl

${ }^{5}$ Ingeniera Forestal. M.Sc. Instituto Forestal, sede Biobío. mmolina@infor.cl
} 


\section{INTRODUCCIÓN}

Los huertos semilleros constituyen uno de los enlaces más importantes que conectan a las poblaciones de mejora con las poblaciones de producción forestal, permitiendo traspasar las ganancias genéticas del programa de mejoramiento para hacerlas efectivas como incrementos de productividad en las plantaciones operacionales (Prescher, 2007). Estas unidades se definen tradicionalmente como plantaciones de clones o de progenies selectas, que se aíslan para minimizar la polinización desde fuentes de menor valor, y se manejan intensivamente para producir semilla en forma frecuente, abundante, fácil de cosechar y con la mayor ganancia genética en el menor tiempo posible (Zobel y Talbert, 1988).

Como tales, los huertos semilleros resultan unidades clave en la producción operacional de material genéticamente mejorado y así se ha entendido también en las iniciativas de mejoramiento de Nothofagus y otras especies nativas emprendidas por el Instituto Forestal en colaboración con la Corporación Nacional Forestal. En este contexto se ha desarrollado, desde 1997, un conjunto de proyectos de investigación, con financiamiento de FONDEF e INNOVA Chile, que han permitido materializar estrategias de mejoramiento genético para especies forestales nativas. Tales proyectos consideraron la selección de árboles plus, la habilitación de fuentes de semilla mejorada (áreas productoras de semillas y huertos semilleros clonales) y la instalación de extensas y muy completas baterías de ensayos genéticos, principalmente pruebas de progenies y procedencias de individuos selectos o representativos de la variabilidad intraespecífica de distintas especies (roble, raulí lenga, coigüe y laurel) a lo largo de su distribución natural (Ipinza et al., 1998).

En la actualidad, una nueva iniciativa de investigación ${ }^{6}$ vuelve a vincular a INFOR y CONAF, ahora para establecer un huerto semillero clonal de segunda generación de roble, derivado de selecciones individuales efectuadas en las pruebas de progenies anteriormente establecidas con esta especie. En términos de propagación y silvicultura de establecimiento, esta nueva iniciativa no difiere significativamente respecto de un huerto de primera generación; sin embargo, en términos de la selección del material a considerar y del diseño de un huerto de segunda generación y siguientes, se requiere tomar decisiones que demandan un rigor técnico considerablemente mayor que para un huerto 1.0 establecido con selecciones masales.

El complejo diseño de los huertos de generación avanzada, con selecciones efectuadas en ensayos genéticos, deriva del interés por usar información cuantitativa (valores de mejora e índices de selección) cuya complejidad se incrementa en la medida que avanzan las generaciones, se mezclan individuos seleccionados hacia adelante y hacia atrás, y se entreveran las relaciones de parentesco entre los árboles evaluados. Por otra parte, la necesidad de maximizar ganancias debe armonizarse con requerimientos de variabilidad genética y reducción de endogamia, aspectos que deben reflejarse en decisiones de diseño tan fundamentales como número de clones a utilizar, cantidad de rametos que represente a cada clon y sus esquemas de distribución espacial.

Consecuentemente, en este artículo se discuten consideraciones claves para enfrentar las decisiones involucradas en el diseño de un huerto de generación avanzada, aspecto que se complementa con antecedentes específicos derivados del trabajo efectuado para diseñar, establecer y en el futuro manejar un huerto semillero clonal de roble (Nothofagus obliqua), de segunda generación. Este huerto en particular se estableció en julio-agosto de 2016, en el predio Huillilemu, a partir de réplicas injertadas de árboles seleccionados en una prueba de progenies de 15 años de edad, en función de sus valores de mejora, los que a su vez fueron estimados mediante técnicas de genética cuantitativa.

En tal contexto, el objetivo de este artículo es documentar el proceso de diseño y establecimiento de un huerto semillero clonal de segunda generación, de modo de constituir una referencia $u$ orientación para otras iniciativas similares que buscan maximizar las ganancias genéticas y minimizar la endogamia bajo restricciones de parentesco.

\footnotetext{
${ }^{6}$ Proyecto CONICYT "Implementación de la Segunda Generación para el Mejoramiento y la Conservación de los Recursos Genéticos de Roble y Raulí: Promoviendo supervivencia, adaptación y potencial económico bajo el escenario de Cambio Climático Global"
} 


\section{HUERTOS SEMILLEROS DE ESPECIES DE Nothofagus EN CHILE}

En el programa de mejoramiento genético de especies de Nothofagus, establecido por Ipinza et al. (1998), se reconoce el potencial productivo de estas especies y se plantea el establecimiento de huertos semilleros clonales como una de sus metas fundamentales para incrementar la productividad de las plantaciones establecidas con estas especies. En este contexto, en el país actualmente existen dos huertos semilleros clonales de $N$. alpina, el primero de ellos, Huillilemu, establecido por la Corporación Nacional Forestal en 1989, en la comuna de San José de la Mariquina en la región de Los Ríos, y el huerto Dewiñ Mahuida establecido en 1999 por la Universidad Austral de Chile y el Instituto Forestal, en convenio con la empresa Forestal Neltume Carranco SA, en la localidad de Neltume, también en la región de Los Ríos.

El huerto Huillilemu fue establecido con un diseño de 71 bloques completos al azar, lo que permite probar diferentes tratamientos con propósito de investigación. Cada bloque contiene 25 clones representados por un rameto cada uno. Esta cantidad de clones permite maximizar la flexibilidad de la depuración y asegurar la remoción del $50 \%$ de los peores clones. La superficie efectiva de huerto corresponde a 2,5 ha. Este huerto aún no posee ensayos genéticos de progenie, aunque para la temporada 2018, se tiene planificado establecer cuatro ensayos de progenie, uno para cada región de procedencia de la región de los Ríos.

El diseño del huerto Dewiñ Mahuida corresponde a un diseño aleatorio con restricción de distancia. Este tipo de diseño es utilizado con fines de producción. La superficie del huerto totaliza 3 hectáreas y se representan en él 24 clones correspondientes a copias vegetativas de árboles plus identificados durante las campañas de selección de árboles superiores. Este huerto se estableció como un gran bloque de 35 filas por 24 columnas, donde los rametos fueron distribuidos al azar considerando una restricción de distancia de 40 metros lineales entre rametos del mismo clon. Este mínimo fue determinado por estudios de distribución de polen alrededor de árboles aislados de coníferas y eucalipto, donde se demostró que la mayor parte del polen cae dentro de esa distancia, o es transportado dentro de ella por los vectores de polinización. Para muchas especies se necesitan estudios de vuelo del polen o el patrón de movimiento de insectos.

El espaciamiento inicial entre rametos, es de $5 \times 5 \mathrm{~m}$ en el huerto Huillilemu y de $6 \times 6 \mathrm{~m}$ en el caso de Dewiñ Mahuida. Este espaciamiento permite un desarrollo adecuado de la copa, como también el acceso de maquinaria para las diferentes labores de manejo; además es utilizado en muchos huertos de polinización abierta de diferentes especies en todo el mundo (Faulkner, 1975).

\section{DISEÑO DE HUERTOS CLONALES DE GENERACIÓN AVANZADA}

La transición desde los huertos semilleros de primera generación hacia los de generación avanzada requiere que se consideren diseños alternativos que permitan maximizar el valor genético de las futuras cosechas de semillas, demostrándose que los diseños aleatorios, que se han usado tradicionalmente en los huertos de primera generación, no siempre resultan eficientes para aprovechar el potencial de las selecciones de generación avanzada (El-Kassaby, 2003).

Para diseñar huertos semilleros apropiados para las generaciones de mejora avanzada se debe tener en consideración algunos criterios particularmente pertinentes, como: la tasa de polinización cruzada; el interés por realizar cruces entre algunos padres específicos; la integración de árboles relacionados (emparentados), tanto de la misma, como de diferentes generaciones; la forma y configuración del huerto; el despliegue proporcional de rametos por clon; y otros. También deben considerarse el rendimiento y los patrones reproductivos de las especies y condiciones ambientales como la dirección predominante de los vientos.

En sus estados iniciales los programas de mejora genética operan con árboles que han sido seleccionados masalmente en rodales silvestres y que no están emparentados entre sí. En esta situación, huertos relativamente simples pueden cumplir eficientemente con la labor de producir semilla mejorada. La literatura ha considerado un sinnúmero de distribuciones espaciales de los huertos semilleros, en especial durante la fase inicial de los programas de mejoramiento genéticos (Giertych, 1975), estos arreglos van desde filas puras, de tablero de ajedrez, 
completamente al azar, bloques completamente al azar, bloques fijados, bloques rotativos, bloques reversados, bloques incompletamente desbalanceados, bloques incompletamente balanceados, bloques incompletos cíclicos, lattice balanceados, vecindad permutada y diseños sistemáticos.

Sin embargo, en la medida que los programas progresan, aumenta la afinidad y parentesco entre los individuos selectos, por lo que surgen nuevas ideas para estructurar los huertos semilleros clonales de generaciones avanzadas, entre ellas usar un número de rametos por clon proporcional a los valores de mejora de cada clon, para así maximizar ganancia al mismo tiempo que se gestiona la diversidad genética, y filas monoclonales, que sin un aumento considerable de endogamia facilitan la cosecha de semillas a nivel de clones individuales.

En general se prefieren los diseños al azar para evitar patrones repetidos de proximidad en ciertos grupos clonales, como ocurre en los diseños sistemáticos. Los patrones de vecindades repetidas son indeseables porque, al momento del raleo genético o depuración de los clones más pobres, se pueden encontrar grupos de clones muy buenos o muy malos que producirán un espaciamiento irregular después del raleo. Cuando hay grupos de clones buenos, puede ser necesario remover algunos rametos simplemente para dejar entrar la luz. Esto ocurre con menos frecuencia en los diseños al azar. Para este diseño se requiere un programa de computador que revise sistemáticamente la restricción de distancia. En programas comerciales de Pinus radiata, en Chile, en la generación 1.5 se ha utilizado normalmente este diseño (Ipinza et al., 1993).

Lstibůrek y El-Kassaby (2007) proponen dos modelos de huertos especialmente útiles para generaciones avanzadas; el primero aprovecha las ventajas de la aleatorización y ordenamiento sistemático de vecindades de clones que comparten padres, para facilitar la cosecha de semillas a nivel de clon (modelo RRSCR ${ }^{7}$ ); y el uso de una asignación global de los rametos de cada clon dentro del espacio total del huerto, con el objeto de minimizar el efecto potencial de la endogamia en la colecta de semillas (modelo de asignación global).

El primer diseño (RRSCR) permite que las filas de cada clon queden separadas por un radio predeterminado, minimizando así la cruza entre los rametos del mismo clon de una fila específica, y maximizando el cruzamiento entre los clones no relacionadas mediante dos elementos de diseño:

(i) Usando filas escalonadas (traslapadas), donde cada clon está rodeado por otros cuatro clones vecinos.

(ii) Usando un conjunto de 4 clones que sean distintos en cada réplica del huerto. Este diseño es relativamente simple, facilita la cosecha selectiva de la semilla por clones individuales y reduce la tasa efectiva de autofecundación y de cruzamiento entre árboles emparentados (Figura $N^{\circ} 1$ ).

Por otra parte, el modelo de asignación global corresponde, en teoría, al diseño más eficiente para reducir la endogamia en la producción de semillas de un huerto. En este esquema la ubicación de cada rameto de cada clon se designa en función de la ubicación de todos los demás. Así, aunque en ocasiones se pueda percibir cierta proximidad entre rametos del mismo clon, este diseño es más efectivo para reducir la endogamia que cualquiera de los otros diseños disponibles (Figura $\mathrm{N}^{\circ}$ 2).

El-Kassaby (2003) comparó huertos de Tsuga heterophylla establecidos con diseños de filas clonales y de asignación global, concluyendo que el diseño no tiene efecto sobre la cantidad de semilla producida; pero que si afecta significativamente a la tasa de exocruzas, indicando que la autopolinización y las cruzas entre individuos relacionados son mayores en el diseño de filas clonales. Esta situación define una considerable diferencia de calidad genética de las semillas a favor del huerto aleatorizado de asignación global.

${ }^{7}$ RRSCR sigla en inglés de: filas clonales, escalonadas (traslapadas), repetidas y aleatorizadas 


\begin{tabular}{|c|c|c|c|c|c|c|c|c|c|c|c|c|c|c|c|c|c|c|c|c|c|c|c|c|c|c|}
\hline & A & B & C & D & $E$ & $F$ & G & $\mathrm{H}$ & 1 & $J$ & K & L & $M$ & $\mathrm{~N}$ & 0 & $P$ & 0 & R & S & $\mathrm{T}$ & $U$ & V & $W$ & $x$ & $Y$ & $z$ \\
\hline 1 & 23 & 7 & 25 & 19 & 8 & 5 & 6 & 22 & 27 & 8 & 1 & 25 & 21 & 22 & 13 & 14 & 1 & 28 & 5 & 13 & 10 & 23 & 22 & 15 & 6 & \\
\hline 2 & 23 & 7 & 25 & 19 & 8 & 5 & 6 & 22 & 27 & 8 & 1 & 25 & 21 & 22 & 13 & 14 & 1 & 28 & 5 & 13 & 10 & 23 & 22 & 15 & 6 & 7 \\
\hline 3 & 23 & (7) & 25 & 19 & 8 & 5 & 6 & 22 & 27 & 8 & 1 & 25 & 21 & 22 & 13 & 14 & 1 & 28 & 5 & 13 & 10 & 23 & 22 & 15 & 6 & 7 \\
\hline 4 & 23 & $Y_{4}$ & 25 & 16 & 8 & 1 & 6 & 13 & 27 & 19 & 1 & 9 & 21 & 11 & 13 & 18 & 1 & 4 & 5 & 9 & 10 & 27 & 22 & 14 & 6 & 10 \\
\hline 5 & 23 & 14 & 25 & 16 & 8 & 1 & 6 & 13 & 27 & 19 & 1 & 9 & 21 & 11 & 13 & 18 & 1 & 4 & 5 & 9 & 10 & 27 & 22 & 14 & 6 & Yo \\
\hline$\underline{6}$ & 23 & 14 & 25 & 16 & 8 & 1 & 6 & 13 & 27 & 19 & 1 & 9 & 21 & 11 & 13 & 18 & 1 & 4 & 5 & 9 & 10 & 27 & 22 & 14 & 6 & 10 \\
\hline 7 & 18 & 14 & 28 & 16 & 12 & 1 & 5 & 13 & 11 & 19 & 24 & 9 & 6 & 11 & 2 & 18 & 26 & 4 & 11 & 9 & 21 & 27 & 4 & 14 & 2 & 10 \\
\hline 8 & 18 & 14 & 28 & 16 & 12 & 1 & 5 & 13 & 11 & 19 & 24 & 9 & 6 & 11 & 2 & 18 & 26 & 4 & 11 & 9 & 21 & 27 & 4 & 14 & 2 & 10 \\
\hline 9 & 18 & 14 & 28 & 16 & 12 & 1 & 5 & 13 & 11 & 19 & 24 & 9 & 6 & 11 & 2 & 18 & 26 & 4 & 11 & 9 & 21 & 27 & 4 & 14 & 2 & 10 \\
\hline 10 & 18 & 4 & 28 & 22 & 12 & 9 & 5 & 28 & 11 & 4 & 24 & 3 & 6 & 14 & 2 & 27 & 26 & 20 & 11 & 22 & 21 & 17 & 4 & 28 & 2 & 25 \\
\hline 11 & 18 & 4 & 28 & 22 & 12 & 9 & 5 & 28 & 11 & 4 & 24 & 3 & 6 & 14 & 2 & 27 & 26 & 20 & 11 & 22 & 21 & 17 & 4 & 28 & 2 & 25 \\
\hline 12 & 18 & 4 & 28 & 22 & 12 & 9 & 5 & 28 & 11 & 4 & 24 & 3 & 6 & 14 & 2 & 27 & 26 & 20 & 11 & 22 & 21 & 17 & 4 & 28 & 2 & 25 \\
\hline 13 & 10 & 4 & 26 & 22 & 15 & 9 & 18 & 28 & 2 & 4 & 25 & 3 & 20 & 14 & & 27 & 3 & 20 & 14 & 22 & 15 & 17 & 6 & 28 & 13 & 25 \\
\hline 14 & 10 & 4 & 26 & 22 & 15 & 9 & 18 & 28 & 2 & 4 & 25 & 3 & 20 & 14 & 7 & 27 & 3 & 20 & 14 & 22 & 15 & 17 & 6 & 28 & 13 & 25 \\
\hline 15 & 10 & 4 & 26 & 22 & 15 & 9 & 18 & 28 & 2 & 4 & 25 & 3 & 20 & 14 & 7 & 27 & 3 & 20 & 14 & 22 & 15 & 17 & 6 & 28 & 13 & 25 \\
\hline 16 & 10 & 5 & 26 & 7 & 15 & 17 & 18 & 5 & 2 & 10 & 25 & 23 & 20 & 16 & 7 & 26 & 3 & 17 & 14 & 16 & 15 & 24 & 6 & & 13 & 21 \\
\hline 17 & 10 & 5 & 26 & 7 & 15 & 17 & 18 & 5 & 2 & 10 & 25 & 23 & 20 & 16 & 7 & 26 & 3 & 17 & 14 & 16 & 15 & 24 & 6 & 7 & 13 & 21 \\
\hline 18 & 10 & 5 & 26 & 7 & 15 & 17 & 18 & 5 & 2 & 10 & 25 & 23 & 20 & 16 & 7 & 26 & 3 & 17 & 14 & 16 & 15 & 24 & 6 & 7 & 13 & 21 \\
\hline 19 & 21 & 5 & 20 & 7 & 28 & 17 & 16 & 5 & 11 & 10 & 6 & 23 & 19 & 16 & $Y_{2}$ & 26 & 5 & 17 & 28 & 16 & 21 & 24 & 12 & 7 & 11 & 21 \\
\hline 20 & 21 & 5 & 20 & 7 & 28 & 17 & 16 & 5 & 11 & 10 & 6 & 23 & 19 & 16 & 12 & 26 & 5 & 17 & 28 & 16 & 21 & 24 & 12 & 7 & 11 & 21 \\
\hline 21 & 21 & 5 & 20 & (7) & 28 & 17 & 16 & 5 & 11 & 10 & 6 & 23 & 19 & 16 & 12 & 26 & 5 & 17 & 28 & 16 & 21 & 24 & 12 & (7) & 11 & 21 \\
\hline 22 & 21 & 15 & 20 & $Y_{3}$ & 28 & 14 & 16 & 3 & 11 & 12 & 6 & 8 & 19 & 18 & 12 & 25 & 5 & 20 & 28 & 6 & 21 & 2 & 12 & Y & 11 & 8 \\
\hline 23 & 21 & 15 & 20 & 13 & 28 & 14 & 16 & 3 & 11 & 12 & 6 & 8 & 19 & 18 & 12 & 25 & 5 & 20 & 28 & 6 & 21 & 2 & 12 & 10 & 11 & 8 \\
\hline 24 & 21 & 15 & 20 & 13 & 28 & 14 & 16 & 3 & 11 & 12 & 6 & 8 & 19 & 18 & 12 & 25 & 5 & 20 & 28 & 6 & 21 & 2 & 12 & 10 & 11 & 8 \\
\hline
\end{tabular}

(Fuente: Lstibůrek y El-Kassaby, 2007).

Las marcas rojas muestran la ubicación en particular del clon $n^{\circ} 7$.

Figura $\mathbf{N}^{\circ} 1$

EJEMPLO DE HUERTO SEMILLERO CLONAL CON DISEÑO RRSCR, DONDE SE EVIDENCIA LA SEPARACIÓN ENTRE LAS REPETICIONES DE LAS FILAS CLONALES, Y EL ORDENAMIENTO ESCALONADO (TRASLAPADO) DE ESTAS FILAS

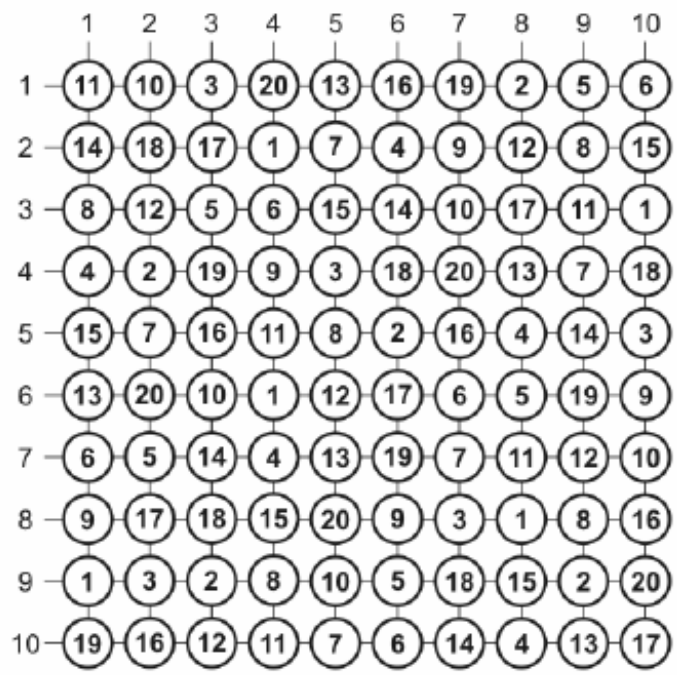

(Fuente: Lstibůrek y El-Kassaby, 2007).

Figura $\mathrm{N}^{\circ} 2$

EJEMPLO DE HUERTO SEMILLERO CLONAL CON DISEÑO DE ASIGNACIÓN GLOBAL, DONDE SE EVIDENCIA LA SEPARACIÓN ENTRE RAMETOS DE UN MISMO CLON. EL EJEMPLO CONSIDERA LA DISTRIBUCIÓN DE 20 CLONES CON 5 RAMETOS CADA UNO. 


\section{ESTABLECIMIENTO DEL HUERTO SEMILLERO CLONAL DE ROBLE EN HUILLILEMU}

\section{Selección e Injertación del Material Genético Considerado en el Huerto}

Los injertos utilizados para conformar el huerto provienen de púas obtenidas desde individuos seleccionados en un ensayo de progenies de roble, de 15 años de edad, previamente establecido en el predio Arquilhue, perteneciente a la empresa Forestal y Agrícola Taquihue SA (región de los Ríos). Tal ensayo fue sometido a una evaluación en la que se midió la altura y DAP de todos sus árboles; con estos datos y el conocimiento de la estructura familiar del ensayo se estimó los valores de mejora para cada individuo mediante un análisis BLUP (Best Linear Unbiased Predictor) con el programa ASREML (Gilmour, et al., 1999). Los árboles seleccionados para injertación, y consecuentemente para conformar el huerto, corresponden a los 22 individuos que presentaron los mayores valores de mejora en diámetro.

La injertación propiamente tal se realizó desde el 15 de julio al 20 de agosto del año 2015, en dependencias del predio Huillilemu. Se utilizó la técnica de hendidura simple, descrita en Emhart et al. (2000), sobre patrones de roble de un año de edad, producidos a raíz desnuda el año 2014 en viveros de Huillilemu.

\section{Emplazamiento y Preparación del Sitio para el Establecimiento del Huerto}

El sitio seleccionado para instalar el huerto se localiza en el predio, en la comuna de San José de la Mariquina en la región de Los Ríos. En la Figura $N^{\circ} 3$ se muestra un fotomosaico del predio, de imágenes captadas por un vehículo aéreo no tripulado, y un detalle del área destinada a establecer el huerto. El manejo de la informacion espacial del fotomosaico se realizo mediante el programa QGIS.
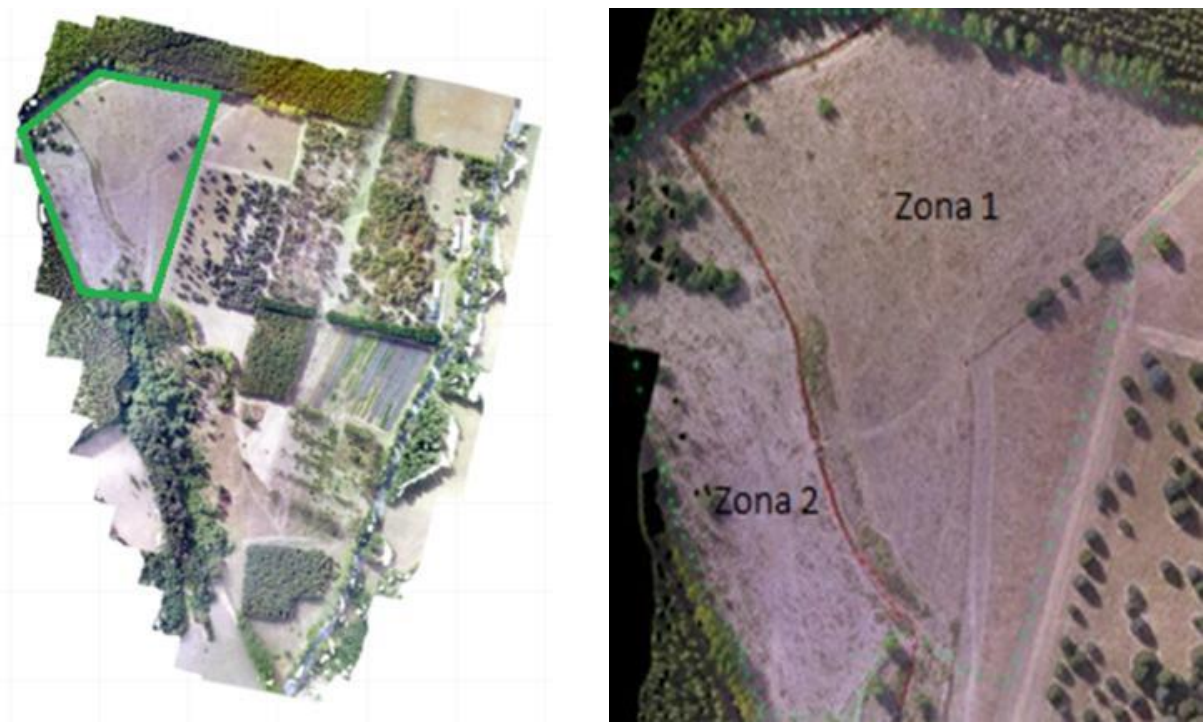

(Fuente: Mosaico realizado por el Dr. Dante Corti)

Zonas 1 y 2 están separadas por un canal de drenaje

Figura $\mathbf{N}^{\circ} 3$

VISTA AÉREA DEL PREDIO HUILLILEMU (IZQ.) Y DETALLE DEL AREA ENMARCADA EN VERDE, QUE CORRESPONDE A LA SUPERFICIE ORIGINALMENTE DISPONIBLE PARA INSTALAR HUERTO SEMILLERO DE ROBLE (DER.) 
El área seleccionada para establecer el huerto corresponde a una superficie aproximada de 3,5 ha, dividida en dos zonas por la presencia de un canal de drenaje que atraviesa el terreno. La zona 2 (1,5 ha) exhibe limitaciones de drenaje, problema que no se presenta en la zona identificada con el número 1 (2,0 ha). En las imágenes de la derecha de las Figuras $\mathrm{N}^{\circ} 3$ y $\mathrm{N}^{\circ} 4 \mathrm{se}$ aprecia el canal de drenaje que separa ambas zonas. La última figura muestra también una vista general del terreno para establecer el huerto.
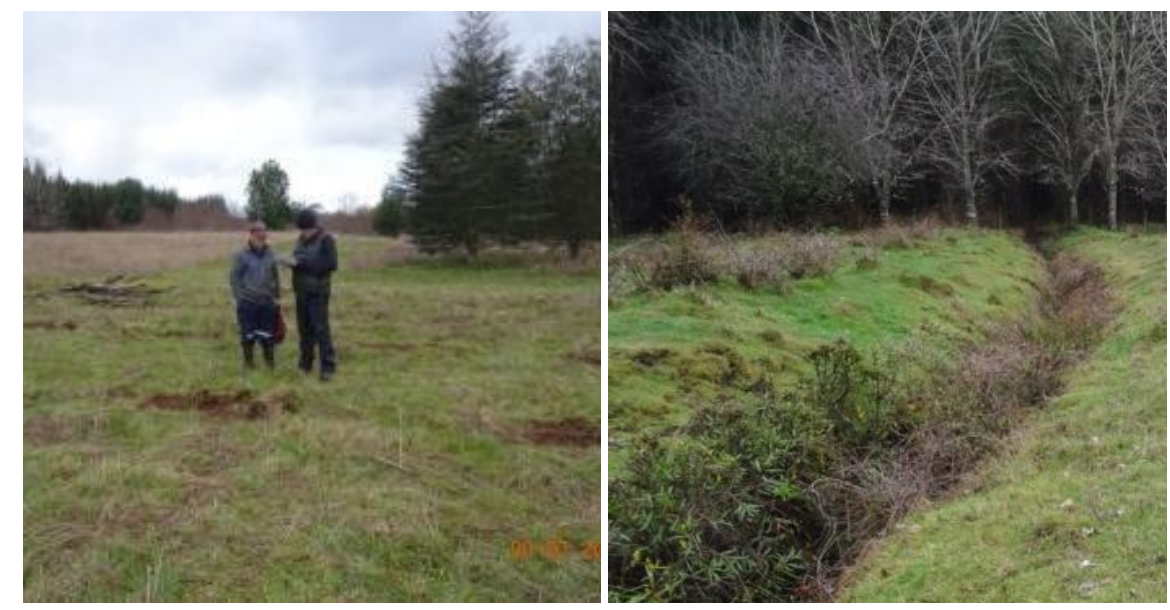

\section{Figura $\mathrm{N}^{\circ} 4$ \\ VISTA GENERAL DEL TERRENO PARA ESTABLECER EL HUERTO (IZQ.) Y DETALLE DEL CANAL DE DRENAJE QUE ATRAVIESA EL TERRENO (DER.)}

La habilitación del terreno destinado al establecimiento del huerto consistió en la remoción de los árboles existentes (principalmente álamos y manzanos) y en la aplicación de una rastra (raneo) para eliminar vegetación indeseable menor (zarzamora y otras malezas).

Una vez realizada la habilitación, se señaló cada posición de plantación disponiendo estacas clavadas en el suelo a un espaciamiento de $5 \times 5 \mathrm{~m}$. En esta operación se excluyó las áreas inundables y con presencia de ripio, así como aquellas que se encontraban a menos de $3 \mathrm{~m}$ con respecto al canal de drenaje. Durante esta actividad se decidió excluir la zona 2, básicamente por los problemas de anegamiento que se presentan, lo que la hace incompatible con la forma de vida que presenta el roble.

El área destinada al huerto de roble (zona 1) es aledaña a un huerto de raulí previamente existente en el predio; así, atendiendo a que ambas especies hibridan, se debió instalar una barrera de polen conformada por una plantación de Pinus radiata y Cupressus spp., dispuesta como cortina de 5 hileras (pino-ciprés-pino-ciprés-pino) a un espaciamiento de $3 \times 3 \mathrm{~m}$ en tresbolillo.

Al considerar el espaciamiento entre rametos $(5 \times 5 \mathrm{~m})$, las superficies no aptas para plantación y el terreno ocupado con la barrera de aislación de polen, se dispone de una superficie útil en la zona 1 para establecer 770 posiciones de plantación.

\section{Diseño del Huerto}

Para formular un diseño de huerto semillero clonal es necesario definir el número de genotipos o clones seleccionados, de modo de armonizar las ganancia y la diversidad genética. También se debe establecer el número de copias o rametos con que se representa cada clon, esto en función de su valor de mejora y del parentesco o relación genética entre los genotipos 
seleccionados; y se debe definir también la distribución espacial de los rametos de cada clon en el terreno. Estas últimas consideraciones son necesarias para reducir la endogamia.

Entonces, para formular un diseño robusto del huerto semillero de roble de Huillilemu, se ha considerado utilizar como una "guía" el programa OPSEL 1.0 (Mullin, 2014) que permite una selección optima de los genotipos, o número de rametos por cada clon, que optimice la ganancia genética. Por su parte, para cumplir con la condición de minimizar la endogamia, en términos de la distribución espacial de los rametos de un mismo clon, se utilizó el programa CLONAGEM (Ipinza et al., 1994). Dicho programa finalmente origina el plano o distribución espacial del huerto semillero diseñado.

En el Cuadro $\mathrm{N}^{\circ} 1$ se resume la información de los clones usados para diseñar y establecer el huerto. En su primera columna muestra un número que indica desde el mejor al peor valor de mejora en DAP; la segunda columna corresponde al código único de los ensayos de progenie y procedencia y de las bases de datos de INFOR; la tercera es el número que corresponde a la familia y el bloque donde se colecto las púas para injerto; la cuarta corresponde al número de rametos sobrevivientes al primero de marzo del 2016. La última columna indica los rametos que son fijos y otros que son comodines.

El total de rametos disponibles para establecer el huerto semillero clonal de roble (Nothofagus obliqua) es de 1.260 , de los cuales 1.140 son considerados fijos (22 clones) y la diferencia (2 clones) son comodines debido a que el número de injertos por rametos es distintos y para prevenir imponderables o accidentes que pueden disminuir el número de rametos por clon de algunos genotipos específicos.

\section{Cuadro $\mathrm{N}^{\circ} 1$ \\ INVENTARIO DE INJERTOS ORDENADOS EN FORMA ASCENDENTE DE ACUERDO A SU VALOR DE MEJORA EN DAP}

\begin{tabular}{|c|c|c|c|c|}
\hline Numero & $\begin{array}{l}\text { Código } \\
\text { Único }\end{array}$ & $\begin{array}{c}\text { Código } \\
\text { Familia + Bloque }\end{array}$ & $N^{\circ}$ de Rametos & $\begin{array}{l}\text { Rametos Fijos } \\
\text { y Comodín }\end{array}$ \\
\hline 1 & 1010 & $479+\mathrm{B} 1$ & 41 & Fijo \\
\hline 2 & 1013 & $423+B 1$ & 36 & Fijo \\
\hline 3 & 1070 & $384+\mathrm{B} 1$ & 57 & Fijo \\
\hline 4 & 1081 & $509+B 1$ & 45 & Fijo \\
\hline 5 & 1245 & $542+B 1$ & 55 & Fijo \\
\hline 6 & 1262 & $341+B 2$ & 52 & Fijo \\
\hline 7 & 1343 & $428+B 2$ & 52 & Fijo \\
\hline 8 & 1491 & $542+B 2$ & 55 & Fijo \\
\hline 9 & 1509 & $514+B 3$ & 27 & Fijo \\
\hline 10 & 1528 & $299+B 3$ & 36 & Fijo \\
\hline 11 & 1579 & $436+B 3$ & 71 & Fijo \\
\hline 12 & 1635 & $380+B 3$ & 62 & Fijo \\
\hline 13 & 1672 & $344+B 3$ & 28 & Fijo \\
\hline 14 & 1763 & $383+B 4$ & 62 & Fijo \\
\hline 15 & 1766 & $472+B 4$ & 76 & Fijo \\
\hline 16 & 1803 & $381+B 4$ & 65 & Fijo \\
\hline 17 & 2028 & $384+B 5$ & 61 & Fijo \\
\hline 18 & 2053 & $455+B 5$ & 34 & Fijo \\
\hline 19 & 2089 & $382+B 5$ & 56 & Fijo \\
\hline 20 & 2146 & $504+B 5$ & 61 & Fijo \\
\hline 21 & 2175 & $457+B 5$ & 78 & Fijo \\
\hline 22 & 2202 & $408+B 5$ & 30 & Fijo \\
\hline 23 & 2208 & $341+B 5$ & 38 & Comodín \\
\hline 24 & 2079 & $503+B 5$ & 82 & Comodín \\
\hline
\end{tabular}




\section{- Maximización de Ganancias Genéticas bajo Restricciones de Parentesco}

En un programa de mejoramiento genético, además de maximizar la respuesta a la selección se debe conservar la diversidad genética (Mullin, 2014). Normalmente no se puede seleccionar el mejor árbol sin tomar en cuenta su grado de parentesco con los demás árboles del huerto. El manejo del parentesco entre selecciones se vuelve más complicado cuando progenitores, hermanos, y otros, tienen un ranking similar. La solución óptima no es evitar completamente la consanguinidad, sino encontrar un conjunto de selecciones que maximicen las ganancias genéticas bajo las restricciones de parentesco.

Para el caso de un huerto semillero de roble, que es de polinización por el viento, Lindgren et al. (1989) y Lindgren y Matheson (1986) sugieren que la relación óptima entre el valor de mejora del candidato y su contribución a la población, es lineal. Esta situación es más probable cuando los candidatos no están relacionados; mientras que si existiera algún tipo de parentesco Bondesson y Lindgren (1993) sugieren soluciones más complejas.

En el Cuadro 2, se muestra los resultados de la aplicación del programa OPSEL, donde se indican los estadígrafos de la solución que converge.

\section{Cuadro $\mathrm{N}^{\circ} 2$}

ESTADÍGRAFOS GENÉTICOS QUE CARACTERIZAN EL NÚMERO DE RAMETOS POR CLON QUE FORM ARA PARTE DEL HUERTO

\begin{tabular}{|l|c|}
\hline Estadígrafos & Valores \\
\hline Número total de entidades en el pedigrí & 1.488 \\
\hline Número de individuos seleccionados & 22 \\
\hline Census number $^{(1)}$ del grupo selecto & 1.140 \\
\hline Status number ${ }^{(2)}$ del grupo selecto & 19.184 \\
\hline Group coancestry $^{(3)}$ del grupo selecto & 0,0260 \\
\hline Self coancestry ${ }^{(4)}$ del grupo selecto & 0,0243 \\
\hline Pair-wise coancestry ${ }^{(5)}$ del grupo selecto & 0,0016 \\
\hline Diversidad genética proporcional del grupo seleccionado & 0,9739 \\
\hline Endogamia promedio del grupo seleccionado & 0,0000 \\
\hline Valor genético promedio del grupo seleccionado & 13,3410 \\
\hline
\end{tabular}

(1) Número total de individuos de una población

(2) Es una medida de diversidad genética y corresponde al número de individuos de una población que no están emparentados

(3) Es el valor promedio de los elementos de la matriz de parentesco. El "Group coancentry" puede dividir en dos partes: "Self coancestry" y "Pair-wise coancestry",

(4) Es la parte dependiente de los ancestros de los genotipos con ellos mismos.

(5) Es la parte dependiente de los ancestros de los diferentes genotipos

El programa arroja además un archivo que indica la nueva relación del número de rametos por clon que maximiza las ganancias genéticas bajo restricciones de parentesco. El parentesco, en el grupo de genotipos seleccionados, 22, es muy bajo $(<0,0001)$, lo cual corresponde a una endogamia promedio de cero.

\section{- Minimización de Endogamia}

De acuerdo con Lstiburek y El-Kassaby (2010) la endogamia en un huerto puede ocasionar pérdida en la producción de semilla debido a la presencia de semillas vanas o de baja tasa de germinación y reducción de las ganancias genéticas en plantaciones forestales. Por esto, en el diseño de un huerto semillero es clave considerar la distribución espacial de los rametos de un mismo clon, para evitar autocruzamiento y así reducir la incidencia de endogamia. En general, a mayor distancia entre rametos del mismo clon menor endogamia. Así, con una distribución espacial 
que mantenga lo más separados posible los rametos de un mismo clon, tal como se muestra en la Figura $\mathrm{N}^{\circ} 5$ (porción del plano final), se logra minimizar la endogamia. Este plano se ha utilizado para la confección del huerto semillero clonal de roble de Huillilemu.

\begin{tabular}{|c|c|c|c|c|c|c|c|c|c|c|c|c|c|c|c|c|}
\hline & 1 & 2 & 3 & 4 & 5 & 6 & 7 & 8 & 9 & 10 & 11 & 12 & 13 & 14 & 15 & \\
\hline 1 & 2053 & 2018 & 1579 & 2146 & 1262 & 1528 & 1635 & 2202 & 1509 & 1803 & 1245 & 2175 & 2089 & 1343 & 1766 & 1 \\
\hline 2 & 1528 & 1013 & 2208 & 1010 & 1491 & 2018 & 1579 & 2146 & 1081 & 1010 & 1579 & 2208 & 2202 & 1672 & 1509 & 2 \\
\hline 3 & 1081 & 2202 & 1766 & 1803 & 1763 & 2175 & 1070 & 1672 & 1013 & 1491 & 1262 & 2018 & 2053 & 1010 & 1245 & 3 \\
\hline 4 & 2089 & 1635 & 1245 & 1343 & 2053 & 1509 & 2208 & 1245 & 1528 & 1635 & 1763 & 1070 & 2146 & 1081 & 1803 & 4 \\
\hline 5 & 1491 & 1070 & 1672 & 1528 & 1013 & 2089 & 1766 & 1343 & 2053 & 2089 & 1766 & 1509 & 1013 & 1635 & 2208 & 5 \\
\hline 6 & 1262 & 1579 & 2146 & 1081 & 2202 & 1262 & 1010 & 1803 & 2018 & 2175 & 1672 & 1245 & 1343 & 1528 & 1763 & 6 \\
\hline 7 & 1803 & 2208 & 1509 & 2018 & 1635 & 1579 & 2146 & 1763 & 2202 & 1579 & 2208 & 1803 & 1491 & 2175 & 1672 & 7 \\
\hline 8 & 2202 & 2053 & 1010 & 1766 & 1070 & 1672 & 1491 & 1013 & 1509 & 1262 & 1010 & 2053 & 2018 & 1579 & 1262 & 8 \\
\hline 9 & 1528 & 1763 & 2089 & 1245 & 1343 & 2175 & 1081 & 1528 & 1635 & 1070 & 2146 & 1081 & 2202 & 1766 & 1010 & 9 \\
\hline 10 & 1081 & 2175 & 1491 & 1803 & 2208 & 2053 & 2089 & 1766 & 1343 & 1245 & 1763 & 1013 & 1635 & 2208 & 2146 & 10 \\
\hline 11 & 1070 & 1262 & 1579 & 2202 & 1013 & 1509 & 2018 & 1010 & 1672 & 1491 & 2089 & 1528 & 1672 & 1070 & 1803 & 11 \\
\hline 12 & 2018 & 2146 & 1672 & 1528 & 1763 & 1262 & 1579 & 2202 & 2208 & 1803 & 1579 & 2175 & 1509 & 1343 & 1081 & 12 \\
\hline 13 & 1509 & 1010 & 2053 & 1635 & 1766 & 1491 & 1245 & 2146 & 1013 & 2053 & 2018 & 1010 & 1262 & 2053 & 1579 & 13 \\
\hline 14 & 2089 & 1013 & 1343 & 1081 & 2175 & 1070 & 1343 & 1635 & 1528 & 1081 & 1766 & 2202 & 1491 & 2089 & 1766 & 14 \\
\hline 15 & 1245 & 2208 & 1803 & 2018 & 2089 & 1672 & 1803 & 1763 & 1262 & 1070 & 1245 & 1635 & 2146 & 1013 & 1528 & 15 \\
\hline 16 & 1528 & 1763 & 2202 & 1579 & 1010 & 2208 & 2053 & 2018 & 2089 & 1509 & 1343 & 1672 & 1763 & 2018 & 1070 & 16 \\
\hline 17 & 1070 & 2175 & 2146 & 1509 & 1262 & 1013 & 2202 & 1579 & 1010 & 1491 & 2208 & 1803 & 1081 & 1509 & 1343 & 17 \\
\hline 18 & 1672 & 1766 & 1635 & 1245 & 1528 & 1766 & 1081 & 2175 & 2146 & 1013 & 2053 & 1262 & 2175 & 1579 & 1245 & 18 \\
\hline 19 & 2053 & 2089 & 1081 & 1491 & 1343 & 1763 & 1070 & 1245 & 1672 & 1766 & 2018 & 2089 & 1010 & 1491 & 2053 & 19 \\
\hline 20 & & 1013 & 2018 & 1803 & 1672 & 2089 & 1635 & 1262 & 1528 & 2202 & 1635 & 1070 & 1528 & 1766 & 2208 & 20 \\
\hline 21 & & & 1010 & 2202 & 2175 & 2053 & 2018 & 2208 & 1509 & 1763 & 1579 & 1343 & 2146 & 1763 & 1262 & 21 \\
\hline 22 & & & & 1509 & 2146 & 1579 & 1013 & 1803 & 1491 & 1010 & 1245 & 1081 & 1803 & 2018 & 1635 & 22 \\
\hline 23 & & & & & 1766 & 1081 & 1343 & 2146 & 2175 & 2053 & 1013 & 1672 & 1509 & 2175 & 1579 & 23 \\
\hline 24 & & & & & 1528 & 1010 & 1763 & 1070 & 1766 & 2089 & 1262 & 2208 & 2202 & 2053 & 1491 & 24 \\
\hline 25 & & & & & & 1672 & 1245 & 1635 & 2202 & 2018 & 1528 & 1635 & 1010 & 1070 & 1013 & 25 \\
\hline 26 & & & & & & & 1509 & 2208 & 1579 & 1343 & 1763 & 2146 & 1766 & 1343 & 1528 & 26 \\
\hline 27 & & & & & & & 1803 & 1013 & 1081 & 1491 & 1803 & 1245 & 2089 & 1262 & 1763 & 27 \\
\hline 28 & & & & & & & & 2175 & 1010 & 1672 & 1070 & 2175 & 2018 & 1081 & & 28 \\
\hline 29 & & & & & & & & & & & & & & & & 29 \\
\hline 30 & & & & & & & & & & & & & & & & 30 \\
\hline
\end{tabular}

Se muestran 15 de las 38 columnas de la zona 1 del huerto, con un número variable de filas. Notar que el rameto con el código único 2053, localizado en las coordenadas $(1,1)$, se vuelve a repetir en las coordenadas $(4,5),(5,9),(8,2),(10,6)$ y en otras.

\section{Figura $\mathbf{N}^{\circ} 5$ \\ PORCIÓN DEL PLANO DEL HUERTO SEMILLERO CLONAL DE ROBLE HUILLILEMU, PLANTADO A UN ESPACIAMIENTO DE 5 X5 METROS.}

Es importante destacar que el diseño final es una mezcla de la información del inventario de los injertos, la biología reproductiva, los valores genéticos, la maximización de las ganancias genéticas bajo restricciones de parentesco, la minimización de la endogamia y la disponibilidad de superficie en el predio Huillilemu.

\section{Plantación del Huerto}

Una vez dispuestas todas las estacas a un espaciamiento de $5 \times 5 \mathrm{~m}$, de acuerdo al diseño establecido, se procedió a trasladar los injertos de cada clon a su respectiva posición de plantación, especificada en el plano del huerto. Durante este procedimiento se verificó rigurosamente que cada rameto se plantara exactamente en el lugar que le correspondía, de acuerdo al plano.

La plantación se realizó en casillas de 40 × 40 y $40 \mathrm{~cm}$, previamente mullidas. Este trabajo se realizó en una superficie de un metro cuadrado que se limpió en forma previa. Después de cada día de plantación se cubrió con "parafilm" la cicatriz de injertación de cada rameto, y se 
rodeó al mismo con un protector de malla raschell (sombra 50\%) de un metro de altura, sostenida por tres estacas de madera alrededor de cada planta. Con esto último se pretende brindar protección para evitar la insolación y proteger a los rametos del ataque de lagomorfos. En la Figura $N^{\circ} 6$ se muestra una secuencia fotográfica de las actividades descritas.
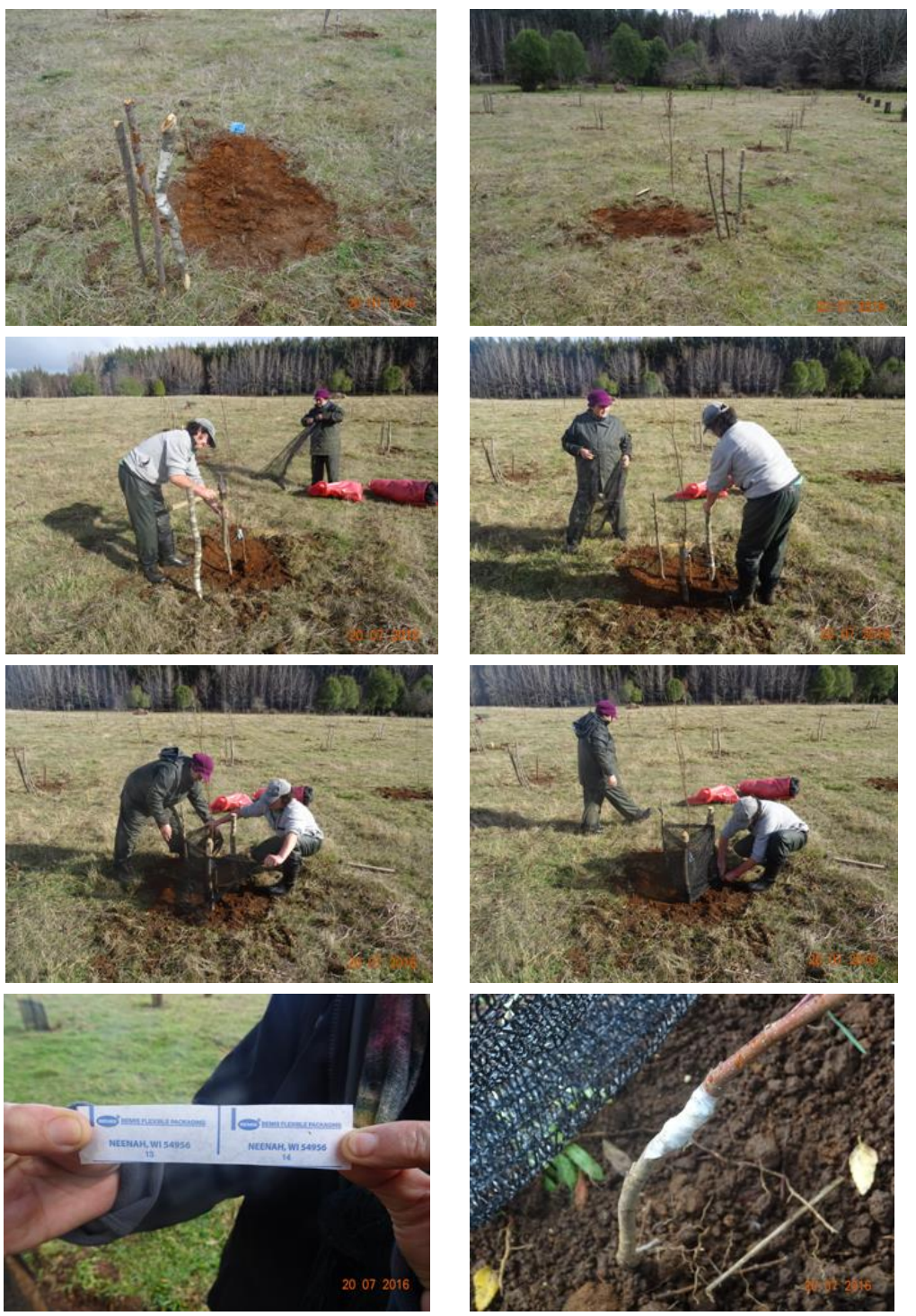

Figura $\mathbf{N}^{\circ} 6$

PREPARACIÓN DE CASILLAS, PLANTACIÓN E INSTALACIÓN DE PROTECTOR DE MALLA RASHELL (6 IMAGENES SUPERIORES)

DETALLE DE ETIQUETA IDENTIFICATORIA DE CADA RAMETO Y DE LA ZONA DE INJERTACIÓN PROTEGIDA CON PARAFILM (2 IMÁGENES INFERIORES) 
Finalmente, en la Figura $N^{\circ} 7$ se muestran imágenes del huerto plantado y del equipo que participó en su plantación.
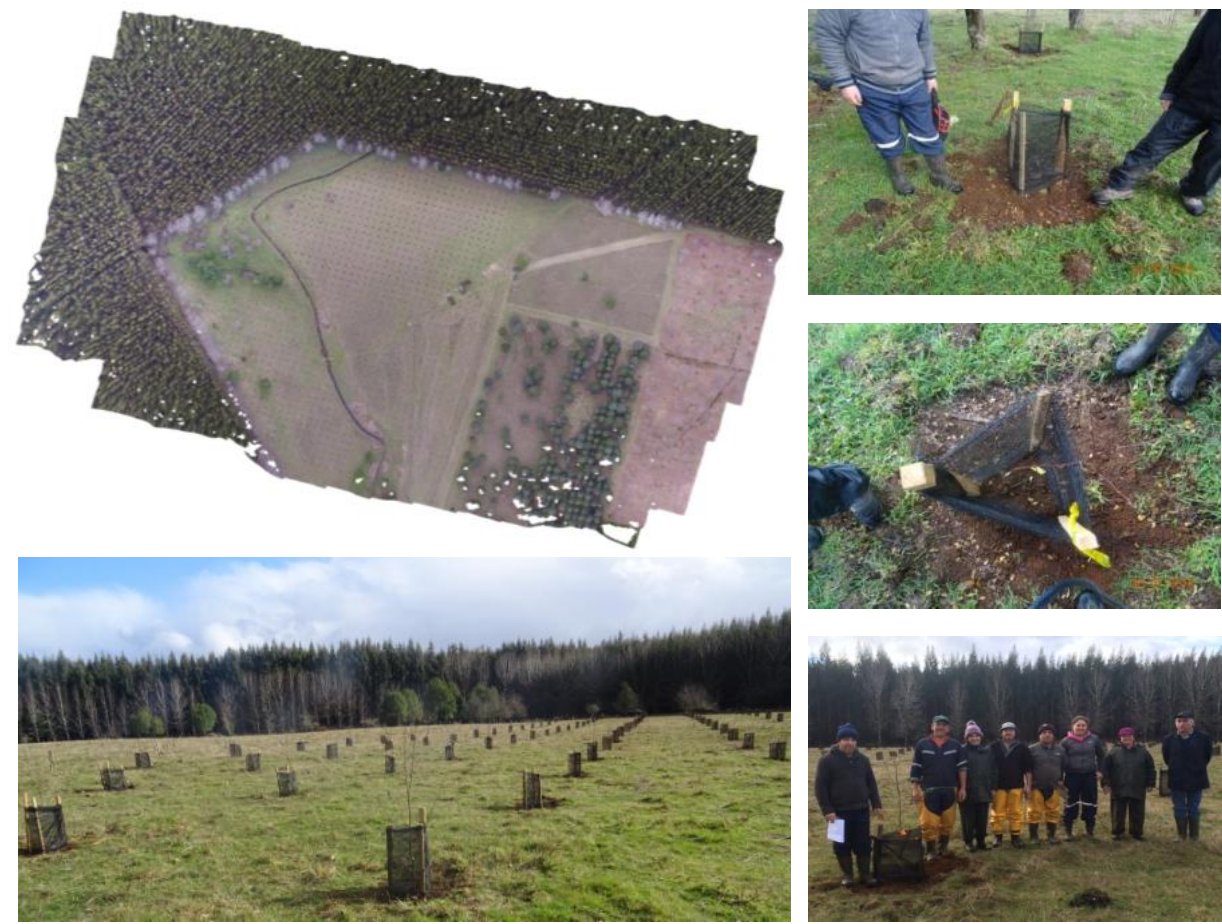

Figura $N^{\circ} 7$

VISTA AÉREA Y ASPECTO GENERAL A NIVEL DEL SUELO DEL HUERTO DE ROBLE ESTABLECIDO EN HUILLILEMU (IZQ.)

DETALLE DE LOS RAMETOS PLANTADOS Y EQUIPO DE TRABAJO QUE REALIZÓ LA PLANTACIÓN (DER.)

\section{Manejo del Huerto}

Para generar una adecuada producción de semillas en el futuro el huerto deberá ser objeto de algunas medidas de manejo. Entre ellas deberán considerarse las siguientes (Granhof, 1991):

\section{Control de Malezas y Manejo de Praderas}

Durante los primeros años del huerto, las plantas deberán mantenerse libres de maleza, ya sea mediante control químico o mecánico. Es aconsejable mantener una cobertura herbácea para prevenir la erosión, mejorar las condiciones para el paso de los equipos y reducir la compactación.

\section{- Riego y Fertilización}

La combinación de riego y fertilización tiene un positivo efecto sobre la producción de flores frutos y semillas, por lo mismo deberá planificarse un esquema regular de fertilización, inicialmente destinado a crecimiento y formación de copa y posteriormente orientado a producción 
de flores. De igual forma se deberá considerar la implementación de medidas que permitan el riego del huerto al menos durante los periodos estivales. Si bien existen antecedentes de que el estrés hídrico podría estimular la producción de flores, no es justificable amenazar la supervivencia ni sanidad de las plantas por falta de agua en periodos críticos de su desarrollo. Se debe estudiar la factibilidad de establecer un "mulch" para mejorar la retención de la humedad alrededor de cada rameto.

\section{Protección}

Las medidas de manejo del huerto deberán considerar proveerle protección contra los posibles daños causados por pisoteo y ramoneo de animales. Deberá considerarse la construcción de cortafuego y la prevención de ataque de insectos $u$ otras plagas y enfermedades. Se debe poner especial cuidado en la mantención de la barrera de polen establecida para minimizar la contaminación de fuentes externas de polen.

\section{Podas y Formación de Copa}

Su función será conformar una estructura de copa simpódica, sin desarrollo monopódico en altura, que favorezca la generación de una abundante ramificación lateral de distintos órdenes donde se desarrollen numerosas yemas florales. Esta operación aumenta la producción de semillas y también facilita la obtención de las mismas

\section{Reguladores de Crecimiento para Inducción Floral}

Una vez que los árboles el huerto alcancen un adecuado desarrollo de sus copas, será conveniente diseñar y aplicar un tratamiento hormonal de inducción floral. Para estos efectos resulta de utilidad utilizar productos basados en paclobutrazol, el que junto con estimular la formación de yemas florales, tiene un efecto secundario reduciendo la elongación de brotes y el crecimiento vegetativo, lo que resulta muy conveniente para evitar el desarrollo en altura de los árboles del huerto, favoreciendo así la colecta de sus semillas. Es posible complementar estas acciones con una adecuada poda apical o topping, que ayudará a promover ramas laterales y finalmente permitirá disminuir la altura del rameto de modo de facilitar labores de cosecha de semilla y de manejo del huerto.

\section{Raleos}

Atendiendo al espaciamiento con que se estableció el huerto, no será necesario efectuar raleos para controlar la competencia entre árboles. Esta intervención se reservará solo para extraer árboles como medida sanitaria y fundamentalmente para depurar el huerto, una vez que se disponga de información de su ensayo de progenies. En esa ocasión el raleo se orientará a eliminar los clones cuyas progenies sean de menor desempeño.

\section{Registros}

Mantener un adecuado registro de la información e historia del huerto es fundamental para orientar su manejo. Es necesario mantener la identidad y localización de cada árbol, el registro de las intervenciones efectuadas y el efecto de las mismas, así como toda la información de interés respecto a floración y producción de semillas en cada temporada.

\section{CONCLUSIONES}

Como resultado del trabajo efectuado se logró establecer un huerto semillero clonal de roble en el predio Huillilemu en la región de Los Ríos. El diseño de este huerto tuvo especial consideración en maximizar las ganancias y diversidad genética, al tiempo que minimiza la endogamia y cruzamiento entre clones emparentados. El huerto consta de 22 clones con un número variable de rametos por clon, que depende del valor de mejora en DAP de cada clon y de su parentesco con los restantes clones del huerto. 
La unidad establecida constituirá una adecuada fuente de semilla mejorada que contribuirá con material genético idóneo para labores de plantación, enriquecimiento, restauración o compensación.

La mantención y manejo futuro del huerto será efectuada conjuntamente por CONAF e INFOR. La colaboración de ambas instituciones ofrece garantías respecto a la pertinencia técnica del manejo del huerto, así como de su cuidado y conservación en el largo plazo.

\section{RECONOCIMIENTOS}

En primer lugar los autores desean agradecer a la asociación gremial APROBOSQUE AG, por su permanente preocupación en pro del desarrollo económico del bosque nativo, así como su esmerada preocupación por mantener y proteger la red de ensayos de progenie y procedencia de roble y raulí establecida el año 2000 , y gracia a lo cual se pudo establecer este huerto de roble de generación avanzada.

La instalación del huerto descrito se realizó gracias a la disponibilidad de superficie en el predio Huillilemu de la Corporación Nacional Forestal, la experiencia del Grupo de Conservación y Mejoramiento Genético del Instituto Forestal y el apoyo y sugerencias del Administrador del predio, Sr. German Clasing. Los autores agradecen al capataz del predio Huillilemu, Sr. Jesús Cofré, por su apoyo en la confección, mantención y cuidados culturales de los injertos empleados para establecer el huerto, así como en la habilitación y supervisión de la plantación de los rametos. Hacen un especial reconocimiento a la Corporación Nacional Forestal por su aporte de insumos y personal del Programa Pro Empleo (PPE). También agradecen al Sr. Rodrigo Sagardía por la colaboración prestada con el software QGIS; y a la Sra. Teresa Parada por su participación en la logística de colecta y traslado de púas para la injertación. MINAGRI.

El análisis genético del ensayo de progenie y procedencia se realizó gracias al apoyo de

\section{REFERENCIAS}

Bondesson, L., and Lindgren, D., 1993. Optimal utilization of clones and genetic thinning of seed orchards. Silvae Genet. 42(4-5): 157-163.

El-Kassaby, Yousry, 2003. Clonal-row vs. Random seed orchard designs: Mating pattern and seed yield of western hemlock (Tsuga heterophylla (Raf.) Sarg.). Forest genetic 10(2): 121-127

Emhart, V.; Hernández, M. y Jofré, J., 2000. Injertación en Nothofagus alpina y N. obliqua. En: Ipinza, R.; Gutiérrez, B. y Emhart, V. (Eds). Domesticación y Mejora Genética de Raulí y Roble. Universidad Austral de Chile, Instituto Forestal. Valdivia. Cap 6, pp: 257-282.

Faulkner, R. , 1975. Seed Orchards. Forestry Commission Bulletin № 54. Faulkner R. (ed). London, England. 149 p.

Giertych, M., 1975. Seed orchard design. In: Faulkner, R (Ed.) Forestry Commission Bulletin $N^{\circ} 54$. England. Pp: 25-33.

Gilmour, A.; Cullis, B.; Welham, S. y Thompson, R., 1999. ASREML. Beta Version. 177 p.

Granhof, J. (recopilador), 1991. Mass production of improved material (2 ${ }^{\text {nd }}$ part). Seed orchards: concepts, design and role in tree improvement. Class Note N D-8. Humlebaek, Denmark. Pp: 19-53.

Ipinza, R.; Perez, E.; Apiolaza, L, y Crespell, P., 1993. Décimo Informe Anual (1993). CMG/UACH/CONAF/Empresas Forestales. Serie Técnica. Universidad Austral de Chile. Facultad de Ciencias Forestales. Valdivia, Chile. $78 \mathrm{p}$.

Ipinza, R.; Perez, E.; Apiolaza, L.; Crespell, P. y Morales, E., 1994. Informe Anual Decimoprimero (1994). CMG/UACH/CONAF/Empresas Forestales. Serie Técnica. Universidad Austral de Chile. Facultad de Ciencias Forestales. Valdivia, Chile. $82 \mathrm{p}$. 
Ipinza, R.; Emhart, V; Gutiérrez, B. y Molina, M., 1998. Mejoramiento Genético para Especies de Nothofagus: Una Pauta Sencilla. Chile Forestal № 257. Enero y febrero 1998.

Lindgren, D., Libby, W. S., and Bondesson, F. L., 1989. Deployment to plantations of numbers and proportions of clones with special emphasis on maximizing gain at a constant diversity. Theor. Appl. Genet. 77(6): 825-831.

Lindgren, D., and Matheson, A. C., 1986. An algorithm for increasing the genetic quality of seed from seed orchards by using the better clones in higher proportions. Silvae Genet. 35(5-6): 173-177.

Lstibůrek, Milan and El-Kassaby, Yousry, 2007. Advanced Generation Seed Orchard Designs. Papers presented at the Seed Orchard Conference. Umea, Sweden. 26-28 Sept, 20072007. En: http://daglindgren.upsc.se/Umea07/Proceedings/LstiburekDe sign.pdf (consulta julio 2016).

Lstibůrek, M. y El-Kassaby, Y., 2010. Minimum-Inbreeding Seed Orchard Design. Forest Science 56(6). 603608.

Mullin, T., 2014. OPSEL 1.0: A computer program for optimal selection in forest tree breeding. Arbets rapport. Från Skogforsk nr. 841-2014. Skogforsk.

Prescher, F., 2007. Seed Orchards: Genetic Considerations on Function, Management and Seed Procurement. Doctoral thesis. Department of Forest Genetics and Plant Physiology. Faculty of Forest Sciences. Swedish University of Agricultural Sciences. Umeå, Sweden.

Zobel, B. y Talbert, J., 1988. Técnicas de Mejoramiento Genético de Árboles Forestales. Editorial Limusa S.A. de C.V. México. $545 \mathrm{p}$. 
